\title{
THE AHLFORS ESTIMATE
}

\author{
JOHN ERIK FORNAESS ${ }^{1}$
}

\begin{abstract}
The Ahlfors estimate gives an upper bound on the growth of a complete Hermitian metric on the punctured unit disc, whose Gaussian curvature is bounded above by -1 . A. Sommese has obtained certain lower bounds on the growth as well. We answer two questions concerning lower bounds, raised by Sommese.
\end{abstract}

The well-known Ahlfors estimate is a basic tool in transcendental algebraic geometry [1] $[3]$.

Proposition 1 (AhIfors estimate). Let $d s^{2}=h(z)|d z|^{2}$ be a complete Hermitian metric on the punctured disc $\Delta^{*}=\{z \in \mathbf{C}: 0<|z|<1\}$. If the Gaussian curvature is bounded above by a negative constant $-b$, then $h<$ $C|z|^{-2}(\ln 1 /|z|)^{-2}$ for a constant $C$ dependent only on $b$.

It is a natural question to ask whether this estimate can be reversed [3], [4]. In [4] Sommese studied the case $h(z)=h(|z|)$ and proved under this additional assumption that for any $\varepsilon>0$ and any $r \in\langle 0,1\rangle$ there exists a constant $C>0$ such that $h(z) \geqslant C|z|^{e-2}$ whenever $0<|z|<r$.

Question 2 (Sommese [4]). Assume $h(|z|)|d z|^{2}$ is a complete Hermitian metric on the punctured disc and suppose that the Gaussian curvature is bounded above by the negative constant $-b$. Does there exist for any $\varepsilon\rangle 0, r \in\langle 0,1\rangle a$ constant $C>0$ such that

$$
h(|z|)>C|z|^{-2}(\ln 1 /|z|)^{-2-\varepsilon}
$$

whenever $0<|z|<r$.

Our main result is that this last estimate fails in general.

Proposition 3. There exists a complete Hermitian metric on $\Delta^{*}, h(|z|)|d z|^{2}$, with Gaussian curvature bounded above by -1 , such that there exists a sequence $\left\{z_{n}\right\}_{n=1}^{\infty} \subset \Delta^{*}, z_{n} \rightarrow 0$ with $h\left(\left|z_{n}\right|\right) \leqslant\left|z_{n}\right|^{-2}\left(\ln 1 /\left|z_{n}\right|\right)^{-n}$.

This example suggests that the result of Sommese mentioned above is the best possible.

In [4], Sommese also asked what kind of lower bounds we have if $h(z)$ is not necessarily radial. We prove here:

Received by the editors July 7, 1978.

AMS (MOS) subject classifications (1970). Primary 53B35, 32H20; Secondary 31A05.

Key words and phrases. Ahlfors lemma.

${ }^{1}$ Research supported in part by an NSF grant at Princeton University. 
Proposition 4. There exists a complete Hermitian metric on $\Delta^{*}, h(z)|d z|^{2}$, with Gaussian curvature bounded above by -1 such that $\lim _{\inf _{z \rightarrow 0}} h(z)=0$.

We are deeply grateful to A. Sommese for having introduced us to this problem and for furnishing essential information.

1. The construction of the metric in Proposition 3 follows an inductive process. We will need the following lemma.

LEMMA 5. Assume $H(t):(-\infty, 0) \rightarrow \mathbf{R}$ is a smooth function such that $H^{\prime \prime}(t) \geqslant \exp (H(t))$ and $\int_{-\infty}^{-1} \exp (H(t) / 2) d t=\infty$. Then $h(z)|d z|^{2}$ is a complete Hermitian metric on the punctured disc, where $h(z)=\exp [H(\ln |z|)-$ $2 \ln |z|]$. The curvature of $(h(z) / 2)|d z|^{2}$ is bounded above by -1 .

Proof. To show completeness, it suffices to verify that $\int_{0}^{1 / 2} \sqrt{h(r)} d r=$ $\infty$. This follows from the identity $\int_{0}^{1 / 2} \sqrt{h(r)} d r=\int_{-\infty}^{-\ln 2} \exp (H(t) / 2) d t$.

The curvature condition is equivalent to the estimate $(\Delta \ln h / 2) / 2>h / 2$ which is equivalent to $H^{\prime \prime}(t) \geqslant \exp (H(t))$. Q.E.D.

To construct the metric we will inductively choose intervals $\left\{\left(a_{n}, b_{n}\right)\right.$, $\left.\left(c_{n}, d_{n}\right)\right\}_{n=1}^{\infty}$ with $a_{n}<b_{n}<c_{n}<d_{n}<a_{n-1} \forall n$ and $d_{1}=-1$ and $\lim _{n \rightarrow \infty} a_{n}$ $=-\infty$. The function $H$ will be constructed to have the following properties:

(i) $\int_{c_{n}}^{d_{n}} \exp (H(t) / 2) d t>1$.

(ii) $)_{n} H(t)<-n \ln |t|$ for at least one point in $\left(a_{n}, b_{n}\right)$.

(iii) $)_{n}$ On $\left(c_{n}, d_{n}\right), H(t)=C_{n}-\left(2+\varepsilon_{n}\right) \cdot \ln |t|$ for some constants $\varepsilon_{n}>0$, $C_{n}$.

(iv) ${ }_{n}$ On $\left(a_{n}, b_{n}\right), H(t)=C_{n}^{\prime}-(n+2) \ln \left|t-\alpha_{n}\right|$ for some constants $\alpha_{n}>$ $0, C_{n}^{\prime}$.

Let us first choose $a_{1}<b_{1}<c_{1}<d_{1}$ and define $H$ on $\left(a_{1}, 0\right)$ to satisfy (i) -(iv) $_{1}$. We choose $C_{1}=0$. Then (i) reduces to $\int_{c_{1}}^{-1}|t|^{-1-e_{1} / 2} d t>1$. Since $\int_{-\infty}^{-1} d t /|t|=\infty$, this can clearly be arranged by choosing $\varepsilon_{1}>0$ sufficiently small and $-c_{1}>0$ sufficiently large. Hence $H(t)=-\left(2+\varepsilon_{1}\right) \ln |t|$ on $\left(c_{1}, 0\right)$ can be assumed to satisfy (i). Also $H^{\prime \prime}(t)=\left(2+\varepsilon_{1}\right)|t|^{-2}>e^{H}=$ $|t|^{-2-e_{1}}$ so we have the right curvature condition on $\left(c_{1}, d_{1}\right)$. (Because of scaling it suffices to have a metric on $\{0<|z|<1 / e\}$, corresponding to $t<d_{1}$.)

We observe that (i) $)_{1}$ and (iii) $)_{1}$ are still satisfied if we increase $\left|c_{1}\right|$ as we do below whenever needed.

To define $\left(a_{1}, b_{1}\right)$ we solve at first the equations

$$
\begin{aligned}
& -\left(2+\varepsilon_{1}\right) \ln \left|c_{1}-1\right|=C_{1}^{\prime}-3 \ln \left|c_{1}-1-\alpha_{1}\right|, \\
& -\left(2+\varepsilon_{1}\right)\left|c_{1}-1\right|^{-1}=-3\left|c_{1}-1-\alpha_{1}\right|^{-1}
\end{aligned}
$$

to obtain $\alpha_{1}=\left|c_{1}-1\right|\left(1-\varepsilon_{1}\right)\left(2+\varepsilon_{1}\right)^{-1}$ and $C_{1}^{\prime}=\ln \left(3 /\left(2+\varepsilon_{1}\right)\right)^{3} \mid c_{1}-$ $\left.1\right|^{1-\varepsilon_{1}}$. With these values, $C_{1}^{\prime}-3 \ln \left|t-\alpha_{1}\right|$ and $-\left(2+\varepsilon_{1}\right) \ln |t|$ have the same values and first derivatives at $t=c_{1}-1$. If we let $\tilde{H}(t)=C_{1}^{\prime}-3 \ln \left|t-\alpha_{1}\right|$ we get that $\tilde{H}^{\prime \prime}(t) / \exp \tilde{H}(t)=3\left|t-\alpha_{1}\right|\left(\left(2+\varepsilon_{1}\right) / 3\right)\left|c_{1}-1\right|^{e_{1}-1}$. Hence if $\left|c_{1}\right|$ 
is sufficiently large, $\tilde{H}^{\prime \prime}(t)>\exp \tilde{H}(t)$ when $t<c_{1}$. We let $H(t)$ extend to the left of $c_{1}-2$ as $\tilde{H}(t)$ and between $c_{1}-2$ and $c_{1}$ we let $H(t)$ be a smoothing of $C_{1}^{\prime}-3 \ln \left|t-\alpha_{1}\right|, t<c_{1}-1$, and $-\left(2+\varepsilon_{1}\right) \ln |t|, t>c_{1}-1$. In this fashion, $H(t)$ can be made smooth and satisfy $H^{\prime \prime}(t)>\exp H(t)$. We choose $a_{1}<b_{1}<c_{1}-2$ such that $H(t)<-\ln |t|$ for some $t \in\left(a_{1}, b_{1}\right)$. This will make $H$ satisfy (i) $)_{1}$-(iv) $)_{1}$.

Assume next that (i) $)_{1}-(\mathrm{iv})_{1}, \ldots,(\mathrm{i})_{n}-(\mathrm{iv})_{n}$ are all satisfied and $H$ is defined on $\left(a_{n}, 0\right)$ and $H^{\prime \prime}(t) / \exp H(t)>1$ on $\left(a_{n},-1\right)$.

Let $d_{n+1}=a_{n}-1$ and consider the equation

$$
C_{n+1}-\left(2+\varepsilon_{n+1}\right) \ln \left|d_{n+1}\right|=C_{n}^{\prime}-(n+2) \ln \left|d_{n+1}-\alpha_{n}\right| \text {. }
$$

We may assume that $-a_{n}>0$ is so large that $\left(2+\varepsilon_{n+1}\right) /\left|d_{n+1}\right|<(n+$ 2) $/\left|d_{n+1}-\alpha_{n}\right|$ for all $0<\varepsilon_{n+1}<\frac{1}{2}$ say. We will find $C_{n+1}$ and $\varepsilon_{n+1}>0$ such that $(\mathbf{i})_{n+1}$ can be satisfied, i.e.,

$$
\int_{c_{n+1}}^{d_{n+1}}\left(e^{C_{n+1} / 2} /|t|^{1+e_{n+1} / 2}\right) d t>1 .
$$

This can be obtained by choosing $\varepsilon_{n+1}>0$ sufficiently small and $-c_{n+1}$ sufficiently large and by choosing $C_{n+1}$ to satisfy the above equation. The condition that $C_{n+1}-\left(2+\varepsilon_{n+1}\right) \ln |t|$ satisfies $H^{\prime \prime} / \exp H>1$ reduces to $\left|d_{n+1}-\alpha_{n}\right|^{n+2}>e^{C_{n}^{\prime}} \cdot\left|d_{n+1}\right|^{2}$ which may be assumed.

We define $H(t)$ on $\left(c_{n+1}, 0\right)$ by smoothing

$$
\max \left\{C_{n+1}-\left(2+\varepsilon_{n+1}\right) \ln |t|, C_{n}^{\prime}-(n+2) \cdot \ln \left|t-\alpha_{n}\right|\right\}
$$

near $d_{n+1}$ and otherwise letting $H(t)$ be the already defined $H$ for $t>d_{n+1}$ and be $C_{n+1}-\left(2+\varepsilon_{n+1}\right) \ln |t|$ on $\left(c_{n+1}, d_{n+1}\right)$.

Let $\alpha_{n+1}=\left|c_{n+1}-1\right|\left(n+1-\varepsilon_{n+1}\right) /\left(2+\varepsilon_{n+1}\right)$ and

$$
C_{n+1}^{\prime}=C_{n+1}+\ln \left(\frac{n+3}{2+\varepsilon_{n+1}}\right)^{n+3} \cdot\left|c_{n+1}-1\right|^{n+1-\varepsilon_{n+1}} .
$$

Then $C_{n+1}^{\prime}-(n+3) \ln \left|t-\alpha_{n+1}\right|$ and $H$ have the same value and slope at $t=c_{n+1}-1$. Moreover, the function $C_{n+1}^{\prime}-(n+3) \ln \left|t-\alpha_{n+1}\right|$ satisfies the equation

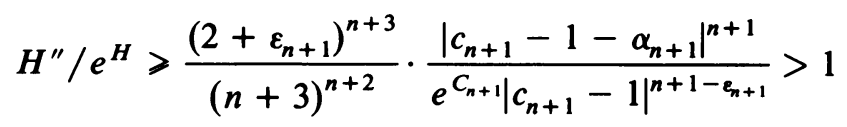

if $-c_{n+1}$ is sufficiently large. Hence we can obtain $a_{n+1}, b_{n+1}$ by the same argument as was used to find $a_{1}, b_{1}$. Therefore we can arrange for $(\mathrm{i})_{n+1^{-}}$ (iv) $)_{n+1}$ to be satisfied and for $H$ to satisfy $H^{\prime \prime}>>\exp H$ on $\left(a_{n+1},-1\right)$.

Completing the induction we obtain a smooth function $H$ defined on $(-\infty, 0)$ which satisfies the curvature condition $H^{\prime \prime}>\exp H$ on $(-\infty,-1)$ and the completeness condition

$$
\int_{-\infty}^{-1} \exp (H / 2) d t \geqslant \sum_{n=1}^{\infty} \int_{c_{n}}^{d_{n}} \exp (H / 2) d t=\infty .
$$

Furthermore, for some point $t_{n} \in\left(a_{n}, b_{n}\right), H\left(t_{n}\right)<-n \ln \left|t_{n}\right|$. 
As in Lemma 5 , define the metric $h(z)|d z|^{2}$ by $h(z)=\exp [H(\ln |z|)-$ $2 \ln |z|]$. Then $h / 2$ is a complete Hermitian metric whose Gaussian curvature is bounded above by -1 . Furthermore, if $z_{n}=e^{t_{n}}$, we obtain $h\left(z_{n}\right)<$ $\left|z_{n}\right|^{-2}\left(\ln 1 /\left|z_{n}\right|\right)^{-n}$.

This proves Proposition 3. Q.E.D.

2. In this section we prove Proposition 4. First, let $h_{0}(z)=1 / z \bar{z} \ln z \bar{z}$. We will modify $h_{0}$ by multiplying with a suitable infinite product. Consider $\phi_{n}=\ln (|z-1 / n| / 2), n=2,3, \ldots$, and let $\phi_{n}^{*}$ be a subharmonic smoothing of $\phi_{n}$ which is sufficiently close to $\phi_{n}$ for the following estimates to hold.

We choose a sufficiently rapidly decreasing sequence $\varepsilon_{n}>0, \varepsilon_{n} \rightarrow 0$, and define $\psi_{n}=\exp \left(\varepsilon_{n} \phi_{n}^{*}\right)$ and let $h(z)=h_{0}(z) \Pi_{n=2}^{\infty} \psi_{n}$.

Then $h(z)|d z|^{2}$ is a smooth Hermitian metric on the punctured unit disc. Moreover, $h(z)$ is complete since the distance from $-\frac{1}{2}$ to any point can be made to be at most 1 less than the same distance measured with $h_{0}$.

To compute the curvature, we have $\Delta \ln h=\Delta \ln h_{0}+\sum_{n=2}^{\infty} \Delta \ln \psi_{n}=4 h_{0}$ $+\sum_{n=2}^{\infty} \varepsilon_{n} \Delta \phi_{n}^{*} \geqslant 4 h_{0}>4 h(z)$. Hence the curvature is bounded above by -4 . On the other hand

$$
h\left(\frac{1}{n}\right)=h_{0}\left(\frac{1}{n}\right) \prod_{k=2}^{\infty} \psi_{k}\left(\frac{1}{n}\right)<n^{2}(\ln n)^{-2} \cdot \exp \left(\varepsilon_{n} \phi_{n}^{*}\left(\frac{1}{n}\right)\right)<\frac{1}{n}
$$

as we clearly can arrange, since $\phi_{n}(1 / n)=-\infty$. Hence $h(1 / n) \rightarrow 0$ as $n \rightarrow \infty$. Q.E.D.

\section{REFERENCES}

1. S. Kobayashi, Hyperbolic manifolds and holomorphic mappings, Pure and Appl. Math., vol. 2, Marcel Dekker, New York, 1970.

2. P. Griffiths, Entire holomorphic mappings in one and several complex variables, Ann. of Math. Studies, no. 85, Princeton Univ. Press, Princeton, N.J., 1976.

3. Differential geometry and complex analysis, Differential Geometry, Proc. Sympos. Pure Math., vol. 27, Part 2, Amer. Math. Soc., Providence, R.I., 1975, pp. 43-64.

4. A. Sommese, Reversing the Ahlfors estimate, Proc. Amer. Math. Soc. 45 (1974), 242-244.

Departmant of Mathematics, Princeton University, Princeton, New Jersey 08540 